\title{
A Finite Model Property for Gödel Modal Logics
}

\author{
Xavier Caicedo ${ }^{1}$, George Metcalfe ${ }^{2}$, Ricardo Rodríguez ${ }^{3}$, and Jonas Rogger ${ }^{2}$ \\ 1 Departamento de Matemáticas, Universidad de los Andes, Bogotá, Colombia \\ xcaicedo@uniandes.edu.co \\ 2 Mathematical Institute, University of Bern, Switzerland \\ \{george.metcalfe, jonas.rogger\}@math.unibe.ch \\ 3 Departamento de Computación, Universidad de Buenos Aires, Argentina \\ ricardo@dc.uba.ar
}

\section{Introduction}

Gödel modal logics combine Kripke frames of modal logics with the semantics of the well-known fuzzy (and intermediate) Gödel logic. These logics, in particular, analogues GK (for "fuzzy" frames) and GKC (for "crisp" frames) of the modal logic K, have been investigated in some detail by Caicedo and Rodríguez [5, 4] and Metcalfe and Olivetti [11, 12. More general approaches, focussing mainly on finite-valued modal logics, have been developed by Fitting [7, 8, Priest [13], and Bou et al. 2]. Multimodal variants of GK have also been proposed as the basis for fuzzy description logics in [10] and (restricting to finite models) [1].

Axiomatizations were obtained for the box and diamond fragments of GK (where the box fragments of $\mathrm{GK}$ and $\mathrm{GK}^{\mathrm{C}}$ coincide) in [5] and for the diamond fragment of $\mathrm{GK}^{\mathrm{C}}$ in [12]. It was subsequently shown in [4] that the full logic GK is axiomatized either by adding the Fischer Servi axioms for intuitionistic modal logic IK (see [6]) to the union of the axioms for both fragments, or by adding the prelinearity axiom for Gödel logic to IK. Decidability of the diamond fragment of GK was established in [5], using the fact that the fragment has the finite model property with respect to its Kripke semantics. This finite model property fails for the box fragment of GK and $\mathrm{GK}^{\mathrm{C}}$ and the diamond fragment of $\mathrm{GK}^{\mathrm{C}}$, but decidability and PSPACE-completeness for these fragments was established in [11, 12] using analytic Gentzen-style proof systems.

The first main contribution of the work reported here is a decidability proof for validity in full GK and $G^{C}$ that makes use of alternative Kripke semantics for these logics admitting the finite model property. The key idea of this new semantics is to restrict evaluations of modal formulas at a world to a particular finite set of truth values. A similar strategy is used to establish decidability, and indeed co-NP-completeness, for the crisp Gödel modal logic $\mathrm{GS}^{\mathrm{C}}$ based on S5 frames where accessibility is an equivalence relation. Moreover, this logic, an extension of the intuitionistic modal logic MIPC of Bull [3] and Prior 14 with prelinearity and a further modal axiom, corresponds exactly to the one-variable fragment of first-order Gödel logic (see 9 ) ${ }^{1}$

\section{Gödel Modal Logics}

Gödel modal logics are defined based on a language $\mathcal{L}_{\square \diamond}$ consisting of a fixed countably infinite set Var of (propositional) variables, denoted $p, q, \ldots$, binary connectives $\rightarrow, \wedge, \vee$, constants $\perp$, $\top$, and unary operators $\square$ and $\diamond$. The set of formulas Fml $\square \diamond$, with arbitrary members denoted $\varphi, \psi, \ldots$ is defined inductively as usual.

\footnotetext{
${ }^{1} \mathrm{~A}$ full paper with the same title as this extended abstract will be presented at WoLLIC 2013 and may be downloaded from www.philosophie.ch/297
} 
We also fix the length of a formula $\varphi$, denoted $\ell(\varphi)$, to be the number of symbols occurring in $\varphi$ and define $\neg \varphi=\varphi \rightarrow \perp$.

The standard semantics of Gödel logic is characterized by the Gödel t-norm min and its residuum $\rightarrow_{\mathrm{G}}$, defined on the real unit interval $[0,1]$ by

$$
x \rightarrow_{\mathrm{G}} y= \begin{cases}y & \text { if } x>y \\ 1 & \text { otherwise. }\end{cases}
$$

The Gödel modal logics GK and GKC are defined semantically as generalizations of the modal logic $\mathrm{K}$ where connectives behave at a given world as in Gödel logic.

A fuzzy Kripke frame is a pair $\mathfrak{F}=\langle W, R\rangle$ where $W$ is a non-empty set of worlds and $R: W \times W \rightarrow[0,1]$ is a binary fuzzy accessibility relation on $W$. If $R x y \in\{0,1\}$ for all $x, y \in W$, then $R$ is called crisp and $\mathfrak{F}$, a crisp Kripke frame. In this case, we often write $R \subseteq W \times W$ and $R x y$ to mean $R x y=1$.

A GK-model is a triple $\mathfrak{M}=\langle W, R, V\rangle$, where $\langle W, R\rangle$ is a fuzzy Kripke frame and $V$ : Var $\times$ $\mathrm{W} \rightarrow[0,1]$ is a mapping, called a valuation, extended to $V: \mathrm{Fml}_{\square} \diamond \times \mathrm{W} \rightarrow[0,1]$ as follows:

$$
\begin{aligned}
V(\perp, x) & =0 \\
V(\top, x) & =1 \\
V(\varphi \rightarrow \psi, x) & =V(\varphi, x) \rightarrow_{\mathrm{G}} V(\psi, x) \\
V(\varphi \wedge \psi, x) & =\min (V(\varphi, x), V(\psi, x)) \\
V(\varphi \vee \psi, x) & =\max (V(\varphi, x), V(\psi, x)) \\
V(\square \varphi, x) & =\inf \left\{R x y \rightarrow_{\mathrm{G}} V(\varphi, y): y \in W\right\} \\
V(\diamond \varphi, x) & =\sup \{\min (R x y, V(\varphi, y)): y \in W\} .
\end{aligned}
$$

A GK ${ }^{\mathrm{C}}$-model satisfies the extra condition that $\langle W, R\rangle$ is a crisp Kripke frame. In this case, the conditions for $\square$ and $\diamond$ may also be read as

$$
\begin{aligned}
& V(\square \varphi, x)=\inf (\{1\} \cup\{V(\varphi, y): R x y\}) \\
& V(\diamond \varphi, x)=\sup (\{0\} \cup\{V(\varphi, y): R x y\}) .
\end{aligned}
$$

A formula $\varphi \in \mathrm{Fml}_{\square \diamond}$ is valid in a GK-model $\mathfrak{M}=\langle W, R, V\rangle$ if $V(\varphi, x)=1$ for all $x \in W$. If $\varphi$ is valid in all $\mathrm{L}$-models for some logic $\mathrm{L}$ (in particular $\mathrm{GK}$ or $\mathrm{GK}^{\mathrm{C}}$ ), then $\varphi$ is said to be $\mathrm{L}$-valid, written $\models_{\mathrm{L}} \varphi$.

Let us agree to call a model finite if its set of worlds is finite, and say that a logic has the finite model property if validity in the logic coincides with validity in all finite models of the logic. In [5, it is shown that the formula $\square \neg \neg p \rightarrow \neg \neg \square p$ is valid in all finite GK models, but not in the infinite crisp model $\langle\mathbb{N}, R, V\rangle$ where $R x y=1$ for all $x, y \in \mathbb{N}$ and $V(p, x)=1 /(x+1)$ for all $x \in \mathbb{N}$. That is, neither $\mathrm{GK}$ nor $\mathrm{GK}^{\mathrm{C}}$ has the finite model property.

\section{A New Semantics and Finite Model Property}

In order for a $\mathrm{GK}^{\mathrm{C}}$-model to render $\varphi=\square \neg \neg p \rightarrow \neg \neg \square p$ invalid at a world $x$, there must be values of $p$ at worlds accessible to $x$ that form an infinite descending sequence tending to but never reaching 0 . This ensures that the infinite model falsifies $\varphi$, but also that no particular world acts as a "witness" to the value of $\square p$. Our strategy is to redefine models to allow 
only a finite number of values at each world that can be taken by box-formulas and diamondformulas. A formula such as $\square p$ can then be "witnessed" at a world where the value of $p$ is merely "sufficiently close" to the value of $\square p$.

Let us define a GFK-model as a quadruple $\mathfrak{M}=\langle W, R, T, V\rangle$, where $\langle W, R, V\rangle$ is a GKmodel and $T: W \rightarrow \mathcal{P}_{<\omega}([0,1])$ is a function from worlds to finite sets of truth values satisfying $\{0,1\} \subseteq T(x) \subseteq[0,1]$ for all $x \in W$. If $\langle W, R, V\rangle$ is also a $\mathrm{GK}^{\mathrm{C}}$-model, then $\mathfrak{M}$ will be called a $\mathrm{GFK}^{\mathrm{C}}$-model.

The GFK-valuation $V$ is extended to formulas using the same clauses for non-modal connectives as for GK-valuations, together with the revised modal connective clauses:

$$
\begin{aligned}
& V(\square \varphi, x)=\max \left\{r \in T(x): r \leq \inf \left\{R x y \rightarrow_{G} V(\varphi, y): y \in W\right\}\right\} \\
& V(\diamond \varphi, x)=\min \{r \in T(x): r \geq \sup \{\min (R x y, V(\varphi, y)): y \in W\}\} .
\end{aligned}
$$

As before, a formula $\varphi \in \mathrm{Fml}_{\square} \diamond$ is valid in a GFK-model $\mathfrak{M}=\langle W, R, T, V\rangle$ if $V(\varphi, x)=1$ for all $x \in W$, written $\mathfrak{M}=_{\text {GFK }} \varphi$.

Observe now that for the formula $\square \neg \neg p \rightarrow \neg \neg \square p$, there are very simple finite GFK ${ }^{\mathrm{C}}$-countermodels: for example, $\mathfrak{M}_{0}=\langle W, R, T, V\rangle$ with $W=\{a\}, R a a=1, T(a)=\{0,1\}$, and $V(p, a)=$ $\frac{1}{2}$. It is easy to see that $V(\neg p, a)=0, R a a \rightarrow_{\mathrm{G}} V(\neg \neg p, a)=1$, and so $V(\square \neg \neg p, a)=1$. Moreover, $V(\square p, a)=0$ (since $\operatorname{Raa} \rightarrow_{\mathrm{G}} V(p, a)=\frac{1}{2}$, and 0 is the next smaller element of $T(a))$; hence $V(\neg \square p, a)=1$ and $V(\neg \neg \square p, a)=0$. So $1=V(\square \neg \neg p, a)>V(\neg \neg \square p, a)=0$ and $\mathfrak{M}_{0} \not \nvdash_{\mathrm{GFK}} \square \neg \neg p \rightarrow \neg \neg \square p$.

Indeed, it can be shown that every formula $\varphi$ that is not GFK-valid (or GFK ${ }^{\mathrm{C}}$-valid) has a finite GFK (respectively, GFK ${ }^{\mathrm{C}}$ ) counter-model of size exponential in the length of $\varphi$. It follows that validity in GFK and GFK ${ }^{C}$ is decidable. Moreover, since validity in GK and $\mathrm{GK}^{\mathrm{C}}$ can be shown to correspond exactly to validity in GFK and GFK ${ }^{\mathrm{C}}$, respectively, decidability follows also for these logics. More precisely, we have established:

Theorem 1. For each $\varphi \in \mathrm{Fml}_{\square \diamond:}$

(a) $\models_{\text {GK }} \varphi$ iff $\models_{\text {GFK }} \varphi$ iff $\varphi$ is valid in all GFK-models $\mathfrak{M}=\langle W, R, T, V\rangle$ satisfying $|W| \leq(\ell(\varphi)+2)^{\ell(\varphi)}$ and $|T(x)| \leq \ell(\varphi)+2$ for all $x \in W$.

(b) $\models_{\mathrm{GKC}^{\mathrm{c}}} \varphi$ iff $\models_{\mathrm{GFKC}} \varphi$ iff $\varphi$ is valid in all $\mathrm{GFK}^{\mathrm{C}}{ }_{-}$models $\mathfrak{M}=\langle W, R, T, V\rangle$ satisfying $|W| \leq(\ell(\varphi)+2)^{\ell(\varphi)}$ and $|T(x)| \leq \ell(\varphi)+2$ for all $x \in W$.

Moreover, validity in $\mathrm{GK}$ and $\mathrm{GK}^{\mathrm{C}}$ is decidable.

\section{A Crisp Gödel S5 Logic}

The crisp Gödel modal logic $\mathrm{GS}^{\mathrm{C}}$ is characterized by validity in $\mathrm{GK}^{\mathrm{C}}$-models where $R$ is an equivalence relation. This logic may also be viewed as the one-variable fragment of first-order Gödel logic (see [9]).

We define a GFS5 ${ }^{\mathrm{C}}$-model as a $\mathrm{GFK}^{\mathrm{C}}$-model $\mathfrak{M}=\langle W, R, T, V\rangle$ such that $\langle W, R, V\rangle$ is a $\mathrm{GSS}^{\mathrm{C}}$ model and also $T(x)=T(y)$ whenever $R x y$ (ensuring that formulas of the form $\square \varphi$ and $\diamond \varphi$ receive the same truth value in all worlds of the same equivalence class). We are then able to show that every non-GFS5 ${ }^{\mathrm{C}}$-valid formula $\varphi$ has a finite $\mathrm{GFS5}{ }^{\mathrm{C}}$-counter-model of size linear in the length of $\varphi$. Since again we are able to establish a correspondence between GFS5 ${ }^{\mathrm{C}}$-validity and $\mathrm{GS} 5^{\mathrm{C}}$-validity, we obtain the following: 
Theorem 2. For each $\varphi \in \mathrm{Fml}_{\square}: \models_{\mathrm{GSS}^{\mathrm{C}}} \varphi$ iff $\models_{\mathrm{GFS}_{5} \mathrm{C}} \varphi$ iff $\varphi$ is valid in all $\mathrm{GFS}^{\mathrm{C}}{ }_{-}$-models $\mathfrak{M}=\langle W, R, T, V\rangle$ where $|W| \leq \ell(\varphi)+2$ and $|T(x)| \leq \ell(\varphi)+2$ for all $x \in W$. Moreover, validity in $\mathrm{GS5}{ }^{\mathrm{C}}$ and the one-variable fragment of first-order Gödel logic is decidable and indeed co-NP-complete.

\section{References}

[1] F. Bobillo, M. Delgado, J. Gómez-Romero, and U. Straccia. Fuzzy description logics under Gödel semantics. International Journal of Approximate Reasoning, 50(3):494-514, 2009.

[2] F. Bou, F. Esteva, L. Godo, and R. Rodríguez. On the minimum many-valued logic over a finite residuated lattice. Journal of Logic and Computation, 21(5):739-790, 2011.

[3] R. A. Bull. MIPC as the formalisation of an intuitionist concept of modality. Journal of Symbolic Logic, 31(4):609-616, 1966.

[4] X. Caicedo and R. Rodríguez. Bi-modal Gödel logic over [0,1]-valued Kripke frames. To appear in Journal of Logic and Computation.

[5] X. Caicedo and R. Rodríguez. Standard Gödel modal logics. Studia Logica, 94(2):189-214, 2010.

[6] G. Fischer Servi. Axiomatizations for some intuitionistic modal logics. Rend. Sem. Mat. Polit de Torino, 42:179-194, 1984.

[7] M. C. Fitting. Many-valued modal logics. Fundamenta Informaticae, 15(3-4):235-254, 1991.

[8] M. C. Fitting. Many-valued modal logics II. Fundamenta Informaticae, 17:55-73, 1992.

[9] P. Hájek. Metamathematics of Fuzzy Logic. Kluwer, Dordrecht, 1998.

[10] P. Hájek. Making fuzzy description logic more general. Fuzzy Sets and Systems, 154(1):1-15, 2005.

[11] G. Metcalfe and N. Olivetti. Proof systems for a Gödel modal logic. In Proceedings of TABLEAUX 2009, volume 5607 of LNAI, pages 265-279. Springer, 2009.

[12] G. Metcalfe and N. Olivetti. Towards a proof theory of Gödel modal logics. Log. Methods Comput. Sci., 7(2):1-27, 2011.

[13] G. Priest. Many-valued modal logics: a simple approach. Review of Symbolic Logic, 1:190-203, 2008.

[14] A. Prior. Time and Modality. Clarendon Press, Oxford, 1957. 